\title{
Solar activity due to magnetic complexity of active regions
}

\author{
Brigitte Schmieder $^{1}$, Cristina Mandrini ${ }^{2}$, Ramesh Chandra $^{1}$, \\ Pascal Démoulin ${ }^{1}$, Tibor Török ${ }^{1}$, Etienne Pariat ${ }^{1}$ and Wahab Uddin ${ }^{3}$ \\ ${ }^{1}$ Observatoire de Paris, LESIA, Meudon, 92195, France \\ email: brigitte.schmieder \\ ${ }^{2}$ IAFE, CONICET-UBA (FCEN), Buenos Aires, Argentina \\ email: mandrini@iafe.uba.ar \\ ${ }^{3}$ ARIES, Nainital, India
}

\begin{abstract}
Active regions (ARs), involved in the Halloween events during October- November 2003 , were the source of unusual activity during the following solar rotation. The flares on 18-20 November 2003 that occur in the AR NOAA10501 were accompanied by coronal mass ejections associated to some particularly geoeffective magnetic clouds.

Our analysis of the magnetic flux and helicity injection revealed that a new emerging bipole and consequent shearing motions continuously energized the region during its disk passage. The stored energy was eventually released through the interaction of the various systems of magnetic loops by several magnetic reconnection events. Active events on November 18 (filament eruptions and CMEs) were originated by shearing motions along a section of the filament channel that injected magnetic helicity with sign opposite to that of the AR. Two homologous flares, that occurred on November 20, were apparently triggered by different mechanisms as inferred from the flare ribbons evolution (filament eruption and CMEs). We studied in detail the behaviour of two North-South oriented filaments on November 20 2003. They merged and split following a process suggestive of 'sling-shot' reconnection between two coronal flux ropes. We successfully tested this scenario in a $3 \mathrm{D}$ MHD simulation that is presented in this paper.
\end{abstract}

Keywords. Active region, magnetic helicity, filament reconnection

\section{Introduction}

It is now widely accepted that three phenomena, flare, CME, and filament eruption, are different observational manifestations of a sudden and violent disruption of the coronal magnetic field, often simply referred to as "solar eruption" (see e.g. Forbes T. G. 2000). To forecast solar activity we need to understand which are the pre-events and the mechanism that trigger and drive eruptions. Accordingly, a large variety of theoretical models for the initiation of eruptions has been proposed in the last decades: tether-cutting model, magnetic break-out model or loss of equilibrium. Multi-wavelength observations are needed to understand the role of flux emergence and shearing motions at the origin of solar eruptions. The large set of data that exist for the events on November 18 and 20, 2003 allow us to do a very detailed analysis.

\section{Observations and Results}

Active region NOAA 10501 was one of the most complex and eruptive regions during the decay phase of solar cycle 23. This AR is the successor of the also very flare productive active region NOAA 10484, as it was named in the previous solar rotation. AR 10501 produced $12 \mathrm{M}$ flares, some of them accompanied by CMEs, from 18 to 20 November, 

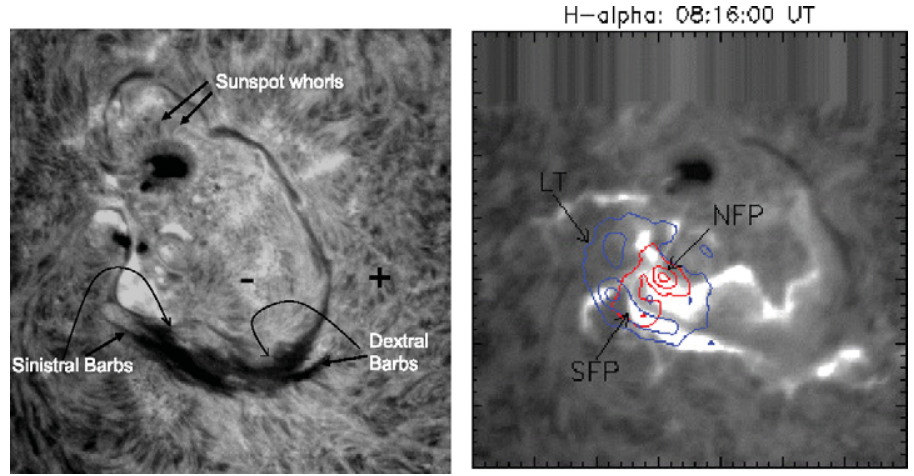

Figure 1. Chromospheric image of AR 10501 on November 18 with arrows pointing to several proxies for the helicity sign (left panel). Two-ribbon flare at 08:16 UT and contours representing RHESSI (25-50 keV) emission (right panel).
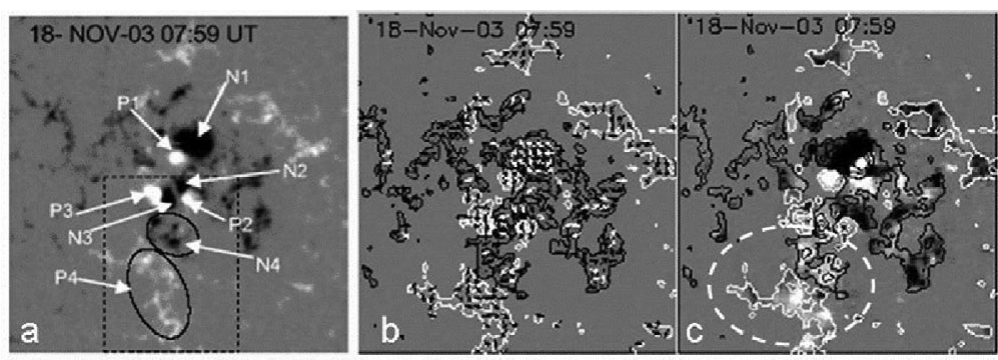

Figure 2. MDI magnetogram of AR 10501 illustrating the different bipoles present on November 18 (a). The same magnetogram with arrows representing the flux transport velocity field (b). Local helicity flux map (c) (white/black indicates positive/negative helicity injection). The oval surrounds a region of positive injection. A portion of a filament lying along this inversion line was ejected; this was probably the source of the magnetic cloud found later in the interplanetary medium.

2003. In particular, the early events on 18 November, 2003, have been extensively studied (Gopalswamy et al. 2005; Yurchyshyn, et al. 2005; Möstl et al. 2008; Srivastava et al. 2009; Chandra et al. 2010a). The peculiarity of this AR on that day was that it presented regions with different magnetic helicity signs, as was discussed by Chandra et al. (2010a).

Chandra et al. (2010a) discussed the multi-wavelength evolution of three solar flares (C3.8, M3.2, M3.9) on 18 November 2003 and concluded that the flares were associated with a filament eruption in the southwest direction which led to a fast CME (Fig 1). The $\mathrm{CME}$ was the source of a very geoeffective magnetic cloud (MC) with positive magnetic helicity on 20 November, 2003. The sign of the magnetic helicity of the active region is opposite to the MC sign.

Several bipoles were seen to emerge as the AR evolved (see Fig 2). Some of these bipoles showed strong rotational motions that injected helicity in the AR (i.e.P3-N3). We speculate that these motions were at the origin of the three flares (one of them shown in Fig 1) observed on November 18 2003. Analyzing the magnetic helicity injection with the method discussed in Pariat et al. (2006), we found that, though the injection had mainly a negative sign in most of the AR, there was a region of positive injection at the location of N4-P4. The topology of this complex active region confirms the existence of several sub-systems of different magnetic helicity sign, one of them is connecting N4-P4 

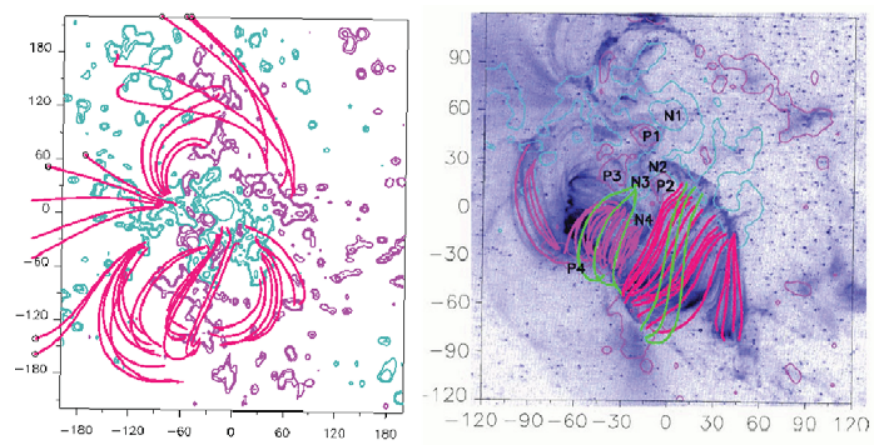

Figure 3. Linear force-free model of AR 10501, all field lines in the left panel have been computed using a negative value for alpha (except those leaving the figure towards the East for which alpha is zero). Field lines in the right panel have been computed using a positive (red and pink) or zero (light green) value for alpha.

(Fig 3). We concluded that the ejection of a portion of the filament lying in between these polarities was the source of the positive helicity MC observed in-situ.

Flux emergence, accompanied by the rotational motion of its polarities, could be also responsible for two homologous flares observed on November 202003 (Chandra et al. 2010b). In the first flare the central inner ribbons were observed first, which can be interpreted in terms of the tether-cutting model for the accompanying eruption (Moore et al. 2001). In the second flare the external ribbons appeared first, which is consistent with the break-out model (Antiochos S. K. 1998). Nearby the flare region two adjacent, elongated north-south oriented filaments merged and separated again; after this process two filaments with different footpoint connections were observed (Figure 4). This process was most probably forced mainly by the rotational motion of one polarity about the other in an emerging bipole at the center of the AR. This evolution was likely to decrease the remnant magnetic field between the two filaments, leading to their approach and interaction. The newly formed southern filament erupted later on, triggering a CME which was the source of an ICME on November 22, 2003.

\section{Interaction of filaments}

Using a 3D zero $\beta$ magnetohydrodynamic (MHD) simulation, Török et al. (2010) showed that the interaction of the two filaments can be explained by "slingshot"
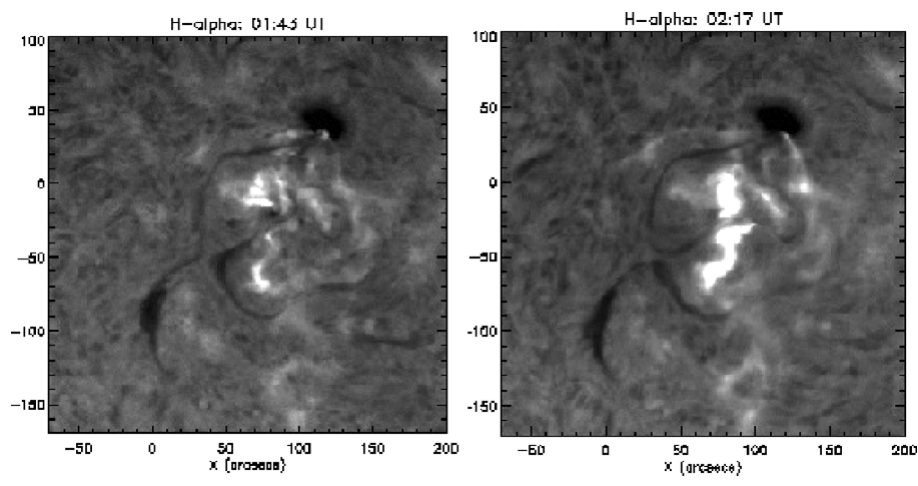

Figure 4. Observation of the interaction of filaments on November 20, 2003 and the inner ribbons of the first flare. 
reconnection between two magnetic flux ropes (Linton et al. 2001). The simulation uses the coronal flux rope model by Titov \& Démoulin (1999) for each filament. The magnetic field between the two flux ropes is first weakened by a diverging flow that moves most of the magnetic flux out of the region between the ropes (Figure 5, top). Then the flux ropes are driven towards each other by converging flows. As they meet, they reconnect and form two new flux ropes with exchanged footpoint connections (Figure 5, bottom). The magnetic dips of the flux ropes (where filament material is assumed to be located) follow this dynamic pattern, in very good agreement with the observed filaments.
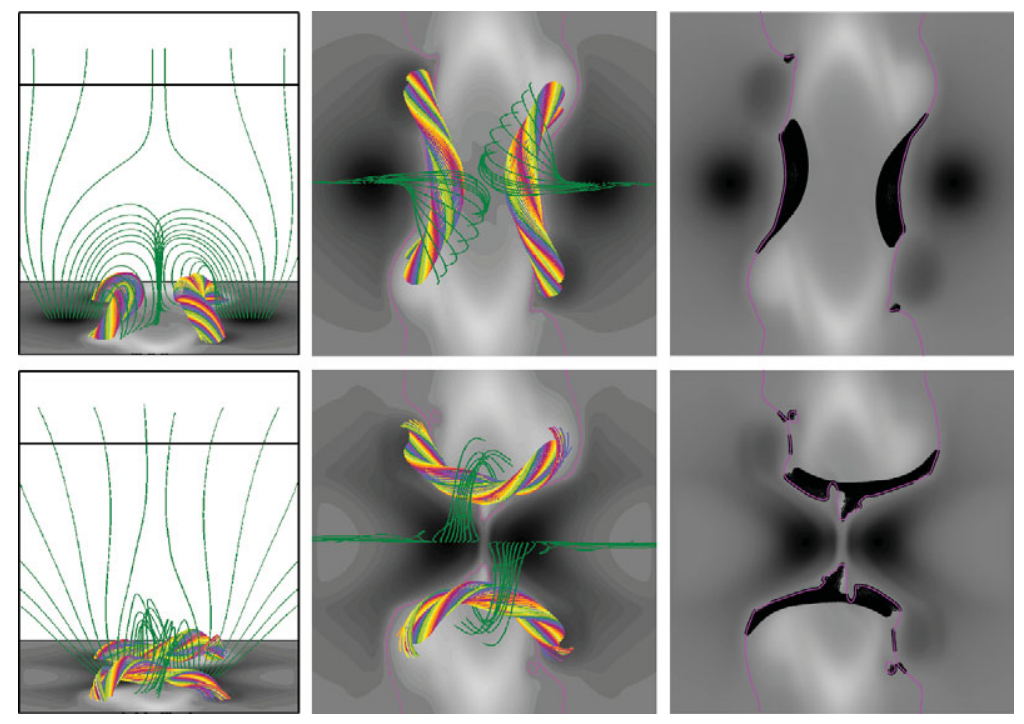

Figure 5. Numerical simulation of reconnection between two magnetic flux ropes by Török et al. (2010)

\section{Conclusion}

The roles of flux emergence and shearing motions are clearly put into evidence in this very complex AR. The stored energy was eventually released through the interaction of the various systems of magnetic loops by several magnetic reconnection events, leading to flares, filament eruption and CMEs.

\section{References}

Antiochos S. K., 1998, Astrophys. J. Lett., 502, 181

Chandra R., Pariat E., Schmieder B., Mandrini C. H., \& Uddin W. 2010a, Solar Phys., 261, 127

Chandra R., Schmieder B., Mandrini C. H., Démoulin P., Pariat E., Török, T., \& Uddin W. 2010b, Solar Phys. (Submitted)

Forbes T. G., 2000, JGR, 105, 23153

Gopalswamy N., Yashiro S., Michalek G., Xie H. Lepping H., \& Howard R. A. 2005, GRL, 32, 12

Linton M. G., Dahlburg R. B., \& Antiochos S. K. 2001, Astrophys. J., 553, 905

Moore R. L., Sterling A. C., Hudson H. S., \& Lemen J. R. 2001, Astrophys. J., 552, 833

Möstl C., Miklenic C., Farrugia C. J., Temmer M., Veronig A. M., Galvin A., Vrsnak B., \& Biernat H. K. 2008, Ann. Geophys, 26, 3139

Pariat E., Nindos A., Démoulin P., \& Berger M. A. 2006, Astron. Astrophys, 452, 623 
Srivastava N., Mathew S. K., Louis R. E., \& Wiegelmann T. 2009, JGR, 114, 3107

Titov V. S. \& Démoulin P. 1999, Astron. Astrophys, 351, 707

Török T., Chandra R., Démoulin P., Schmieder B., Aulanier G., Pariat E., \& Mandrini C. H. 2010, Astrophys. J. (Submitted)

Yurchyshyn V., Hu Q., \& Abramenko V. 2005, Space Weather, 3, 8

\section{Discussion}

GEORGOULIS: What was the instability mechanism that made the two flux ropes merge? Was it flux cancellation in the photosphere or flux higher up that made the two ropes merge?

SCHMIEDER: It's second solution, I think, it's decreasing magnetic field. It can be submergence or dispassion, or that is what we think. 